\title{
Hard Examples for Common Variable Decision Heuristics
}

\author{
Marc Vinyals \\ Computer Science Department, Technion, Haifa 3200003, Israel \\ marcviny@cs.technion.ac.il
}

\begin{abstract}
The CDCL algorithm for SAT is equivalent to the resolution proof system under a few assumptions, one of them being an optimal non-deterministic procedure for choosing the next variable to branch on. In practice this task is left to a variable decision heuristic, and since the so-called VSIDS decision heuristic is considered an integral part of CDCL, whether CDCL with a VSIDS-like heuristic is also equivalent to resolution remained a significant open question.

We give a negative answer by building a family of formulas that have resolution proofs of polynomial size but require exponential time to decide in CDCL with common heuristics such as VMTF, CHB, and certain implementations of VSIDS and LRB.
\end{abstract}

\section{Introduction}

Conflict-Driven Clause Learning (CDCL) is nowadays the top-performing algorithm to solve SAT in practice. When a CDCL solver finds a formula to be unsatisfiable, a proof of this fact can be read from its execution trace, and that proof can be formalized in the resolution proof system (Beame, Kautz, and Sabharwal 2004). It is not obvious whether all resolution proofs can be obtained using a CDCL solver; for instance, the preceding DPLL algorithm is restricted to producing tree-like resolution proofs, which are exponentially weaker than general resolution (Goerdt 1993; Urquhart 1995; Bonet et al. 2000).

Progress towards this question was made showing that tweaked versions of CDCL polynomially simulate resolution-in other words, the algorithm can produce proofs that are only polynomially worse than the optimal resolution proof-either allowing the solver to not resolve conflicts immediately (Beame, Kautz, and Sabharwal 2004) or making an additional pre-processing step (Hertel et al. 2008). The question was largely settled by Pipatsrisawat and Darwiche (2011), who showed that under a few assumptions CDCL polynomially simulates resolution.

Naturally there has been work towards understanding which assumptions are needed; to explain them we have to sketch how the CDCL algorithm works. Similarly to DPLL, the algorithm assigns values to variables, either by

Copyright (c) 2020, Association for the Advancement of Artificial Intelligence (www.aaai.org). All rights reserved. choice or forced by a clause, until either a solution or a conflict is found. Upon reaching a conflict DPLL backtracks and makes the last choice forced to its opposite. CDCL also backtracks, but analyses the conflict to learn a new clause that precludes the same conflict-and potentially many others-from occurring again. Since part of the state is kept in the learned clauses it makes sense to occasionally restart the search, and for memory management reasons to forget some of the clauses.

A first, mild assumption is that the solver restarts often enough. This is a reasonable assumption to make since solvers restart frequently in practice, but it is still interesting to study what would be the case without restarts, if only to understand the power of restarts. The pool resolution subsystem (Van Gelder 2005) captures proofs produced by solvers that do not restart, and a few candidates for a separation between pool and general resolution were proposed but all turned out to have short pool resolution proofs (Bonet, Buss, and Johannsen 2014; Buss and Kołodziejczyk 2014). Long story short, we still do not know if restarts are needed.

Another assumption is that the solver never forgets a clause. This is less realistic since in practice solvers throw out many of the clauses they learn. A much weaker assumption is needed, in that solvers need to keep at least a few selected clauses in order to produce optimal proofs (Elffers et al. 2016), but there is a large stretch between having to keep a few selected clauses and all clauses, and we do not know where in this line the truth lies.

The last assumption we consider is that when the solver chooses which literal to branch on, it makes optimal nondeterministic choices - that is, with external oracle advice. Of course this is not at all what happens in practice, in fact furnishing CDCL with a conflict-based decision heuristic is considered crucial for CDCL to work well if not an inextricable part of CDCL (Marques-Silva, Lynce, and Malik 2009; Katebi, Sakallah, and Marques-Silva 2011; Biere and Fröhlich 2015), hence we cannot close the question whether CDCL polynomially simulates resolution unless we allow a heuristic that is closer to practice.

For instance, we can relax the non-deterministic assumption to frequent enough random choices and obtain a weaker conclusion. In this case, and still using the first two assumptions, CDCL can simulate bounded-width resolution (Atserias, Fichte, and Thurley 2011). At the same time, it is most 
likely that an assumption along these lines is needed, since a recent result shows that if $P \neq N P$ then no deterministic algorithm that produces resolution proofs - in particular CDCL with any deterministic heuristics - can polynomially simulate resolution (Atserias and Müller 2019).

\subsection{Our Results}

In this work we look into this last assumption, nondeterministic branching, and we prove unconditionallywithout depending on whether $\mathrm{P}=\mathrm{NP}$ - that if the CDCL algorithm uses conflict-based heuristics, then it cannot polynomially simulate resolution. This answers in the negative a question of Mikša and Nordström (2014), who ask whether CDCL with VSIDS, 1UIP, and phase saving polynomially simulates resolution.

Theorem 1.1 (Informal). There exists a family of formulas that have polynomial-size resolution proofs but CDCL with conflict-based decision heuristics runs in exponential time.

We explain exactly what we mean with conflict-based in Sections 2.3 and 3, and for now we just say that the decision heuristics we look into all have in common that they select variables that have been recently involved in generating conflicts. These include VMTF, CHB, and certain implementations of VSIDS and LRB.

We do not need to make assumptions on the remaining parts of the algorithm, but it is worth mentioning a caveat concerning which decision heuristics our result applies to. For technical reasons in some heuristics we need to impose that variable scores are stored either with arbitrary precision or in a stable data structure. We opt for the latter choice since, as we argue in Section 2.4, it is a plausible condition that does not make much difference in practice.

In addition to proving a theoretical result, we run experiments on the formulas we craft. The experiments confirm our theoretical findings, that is the formulas are very hard for SAT solvers that employ the heuristics we discuss, but trivial if we specify a static variable order. Even more, for certain parameter ranges it is more effective to make many decisions at random.

\subsection{Related Work}

We know of other branching heuristics that are not enough to simulate resolution; for instance in the context of encoding constraints into $\mathrm{CNF}$ a branching heuristic that only picks input variables (that is variables from the problem domain, as opposed to auxiliary variables introduced during translation to CNF) is exponentially weaker than full CDCL (Järvisalo and Junttila 2009), and recently Mull, Pang, and Razborov (2019) proved that CDCL with ordered decisions and the decision learning scheme is also exponentially weaker than resolution. However, our work is the first to expose the theoretical limits of conflict-based heuristics.

By no means we are the first to observe that there are formulas where VSIDS performs badly or that making random decisions can be helpful-in fact the appendix to the MiniSat solver description (Eén and Sörensson 2004) discusses random decisions, and one may find plenty of concrete ex- amples where a static variable order drastically outperforms VSIDS in the thorough evaluation of Elffers et al. (2018).

\subsection{Techniques}

To prove our result we design a formula with the explicit purpose of tricking decision heuristics. Our formula consists essentially of an easy part that has polynomial-length proofs, a hard part that requires exponential-length proofs, and a set of pitfall gadgets that trick the solver into exploring the hard part before the easy part. Coming up with robust gadgets is a significant part of the work.

Proving an upper bound on resolution length is straightforward, but proving a lower bound on CDCL time becomes a delicate task. To do so we mix a low-level stepby-step simulation with a high-level proof-complexity argument. The simulation is an ad-hoc argument where we run the algorithm for a few steps, carefully controlling the state of the solver, until we reach a state where the solver is restricted to working on a reduced set of variables. Then we use a black-box proof-complexity result to show that any proof of the restricted formula must be of exponential size.

\section{Preliminaries}

\subsection{Proof Complexity}

A resolution refutation of a $\mathrm{CNF}$ formula $F$ is a sequence of disjunctive clauses $C_{1}, \ldots, C_{T}$ such that every clause $C_{t}$ either belongs to $F$ or it can be obtained by applying the resolution rule

$$
\frac{C \vee x \quad D \vee \bar{x}}{C \vee D}
$$

with $C \vee x=C_{i}, D \vee \bar{x}=C_{j}$, and $i, j<t$. The length of a refutation is $T$, the number of clauses in the refutation.

The restriction of a clause $C$ by a truth value assignment $\rho$ is $C \uparrow_{\rho}=\bigvee_{l \in C} \rho(l)$. The restriction of a set of clauses is the result of restricting each individual clause and eliminating tautologies. If $\pi$ is a resolution refutation of $F$, then $\pi \uparrow_{\rho}$ is a valid resolution refutation of $F \uparrow_{\rho}$.

The Tseitin formula of a graph $G(V, E)$ and a charge function $\chi: V \rightarrow\{0,1\}$ is a CNF with one variable per edge and the set of constraints $\operatorname{Ts}(G, \chi)=\bigwedge_{v \in V} \operatorname{Parity}(v)$ where $\operatorname{Parity}(v)$ is the $\mathrm{CNF}$ encoding $\bigoplus_{e \ni v} x_{e}=\chi(v)$. A Tseitin formula is unsatisfiable if and only if $\sum_{v \in V} \chi(v)$ is odd, which we assume from now on. Tseitin formulas are hard to prove within the resolution proof system.

Theorem 2.1 (Urquhart 1987). There is a family of graphs on $n$ vertices and constant degree such that $\operatorname{Ts}(G, \chi)$ requires resolution proofs of length $\exp (\Omega(n))$.

\subsection{The CDCL Algorithm}

We follow the description of CDCL of Elffers et al. (2016). The input is a CNF formula $F$. The state is formed by the trail $\langle\rho\rangle$, which is a sequence of assignments, and the clause database $\mathcal{D}$, which is a set of clauses. Initially $\langle\rho\rangle=\emptyset$ and $\mathcal{D}=F$. The algorithm runs in a loop and the action to take at each step depends on $\mathcal{D} \uparrow_{\rho}$. If the empty clause $\perp$ is in $\mathcal{D} \uparrow_{\rho}$ then we say there is a conflict. If we can resolve it then we learn a clause and add it to $\mathcal{D}$, otherwise the formula 


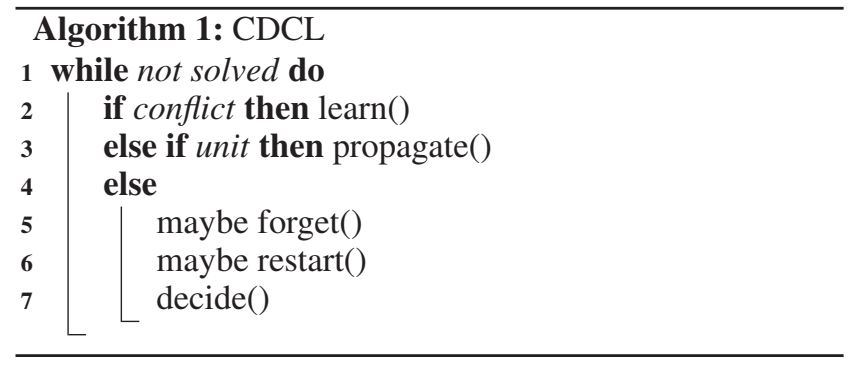

is unsatisfiable. If there is a unit clause $\left.x^{b} \in \mathcal{D}\right|_{\rho}$ then we propagate its value by appending $x=b$ to $\langle\rho\rangle$. Otherwise we say that we are in a stable state. In this case we might forget a few clauses and use a reduced database $\mathcal{D}^{\prime} \subseteq \mathcal{D}$, we might restart the search by resetting the trail to $\langle\rho\rangle=\emptyset$ but keeping the learned clauses, or we decide the value of some unassigned variable by appending $x=b$ to the trail, unless all variables are assigned in which case we found a satisfying assignment.

In order to fully determine the CDCL algorithm we need to specify its learn, propagate, forget, restart, and decide components. We assume about the learning component that any learned clause can be derived from the conflict clause and unit-propagating clauses (Beame, Kautz, and Sabharwal 2004) but nothing more. The clauses used in such derivation are said to participate in a conflict, and so are all the variables contained in clauses that participate in a conflict.

Lemma 2.2 (Beame, Kautz, and Sabharwal 2004). If a formula $F$ over $n$ variables is found unsatisfiable by a CDCL solver in $T$ conflicts, then there is a resolution refutation of $F$ of length at most $n \cdot T$ using only conflict and unitpropagating clauses.

We do not make any assumptions about which variable is propagated first when multiple choices are possible, nor about which clauses are forgotten, nor when to restart the search, and we discuss branching heuristics next.

\subsection{Branching Heuristics}

In the heuristics we consider the decision of which literal to branch on is actually taken by running two procedures, first a variable decision heuristic, or decision heuristic for short, and then a phase decision heuristic. We assume that the phase is chosen according to the phase-saving heuristic, that is with the same sign as the last time that the selected variable was last assigned. If a variable has never been assigned, we assume that it is first assigned to 0 .

The variable decision heuristics we consider all assign a score $q$ to each variable, which is updated as the search progresses, and at the time to make a decision they choose the variable with the largest score. Tie-breaking is often considered an implementation detail.

We consider the top-performing decision heuristics evaluated by Biere and Fröhlich (2015), and in addition two later heuristics that outperform VSIDS on certain benchmarks (Liang et al. 2016a; 2016b).

In VSIDS (Moskewicz et al. 2001; Eén and Sörensson 2004) the score of a variable is bumped each time it par- ticipates in a conflict and decays at every conflict. More formally, the score of variable $x$ after conflict $t$ is $q(x, t)=$ $b(x, t)+\delta \cdot q(x, t-1)$, where $0<\delta<1$ is the decay factor and $b(x, t) \in\{0,1\}$ is 1 if $x$ participated in the conflict. ${ }^{1}$ The decay factor can be a constant or change over time.

In VMTF (Ryan 2004) the score is $q(x, t)=t$ if $x$ participated in the conflict ${ }^{2}$ and $q(x, t-1)$ otherwise. The relative order among tied variables is preserved.

In ACIDS (Biere and Fröhlich 2015) the score is $(t+$ $q(x, t)) / 2$ if $x$ participated in the conflict and $q(x, t-1)$ otherwise.

In CHB (Liang et al. 2016a) the score is updated after every set of propagations leading to a stable state or conflict $s$, but only for variables added to the trail since state $s-1$; the rest is not modified. If variable $x$ is updated at state $s$, then $q(x, s)=\alpha \cdot r(x, s)+(1-\alpha) \cdot q(x, s-1)$, where $r(x, s)=$ $\mu(s) / \Delta(x, s), \mu(s)=1$ if we reached a conflict and 0.9 otherwise, and $\Delta(x, s)$ is the number of conflicts since $x$ last participated in a conflict. The MapleSAT implementation of CHB has $\alpha$ decreasing after each conflict.

In LRB (Liang et al. 2016b) the score is updated after each conflict or restart, but only for variables getting unassigned. We have $q(x, s)$ defined as in $\mathrm{CHB}$, but $r(x, s)=$ $p(x, s) / \Delta(x, s)$, where $p(x, s)$ is the number of times $x$ participated in a conflict or belonged to a clause that propagated a literal found in the learned clause, and $\Delta(x, s)$ is the number of conflicts, all of these measured since $x$ was last assigned. We also decay the score of all variables by a factor $\delta$ after each conflict.

\subsection{Implementation Details}

Precision If we expand the definition of VSIDS we get that $q(x, t)=\sum_{i=1}^{t} \delta^{t-i} b(x, i)$. However, since in practice we keep the scores using floating point numbers, it is more accurate for our model to drop the tail of the sum. In other words we only need to account for the last $K$ conflicts, where $K$ is such that

$$
\sum_{i=1}^{t-K} \delta^{t-i}=\frac{\delta^{K}-\delta^{t}}{1-\delta}<\frac{\delta^{K}}{1-\delta}<\epsilon
$$

with $\epsilon$ being the minimum number that can be represented with a particular floating point type. If we have $e$ bits of exponent, this means

$$
K>\frac{\log (\epsilon(1-\delta))}{\log (\delta)}=\frac{-2^{e}+\log (1-\delta)}{\log (\delta)} .
$$

To put this in numbers, MiniSat uses $e=10$ and $\delta=0.95$, hence no more than the last 14000 conflicts matter.

Asymptotically, if we make the assumption that to solve a problem of size $n$ we use data types of size $\Theta(\log n)$ in bits, then we have $\Theta(\log n)$ bits of floating point exponent,

\footnotetext{
${ }^{1}$ The original Chaff implementation has a score for each literal, a literal is bumped if it belongs to the learned clause, and decays and reordering happen once every 256 conflicts.

${ }^{2}$ The original Siege implementation only bumps variables in the learned clause.
} 
which gives a value for $K$ of $2^{\Theta(\log n)}=n^{\Theta(1)}$, that is only a polynomial number of recent conflicts matter.

In our analysis we need to separate variables that participated in a conflict up to exponentially many conflicts ago from variables that did not participate in a conflict at all, but as we just discussed their scores will be both indistinguishable from 0 , therefore we need to pay attention to tie-breaking. Ultimately which variable gets decided among variables of equal score depends on which data structure is used to implement the priority queue of unassigned variables, hence we consider two kinds of data structures depending on whether they preserve the preexisting order.

A stable priority queue-and, by extension, a stable decision heuristic-has the property that if at a state $s$ we have $q(x, s)>q(y, s)$ and for every state $s^{\prime}>s$ we have $q\left(x, s^{\prime}\right) \geq q\left(y, s^{\prime}\right)$, then $x$ is dequeued before $y$. An example of a stable priority queue is a radix heap (Biere and Fröhlich 2015), while an example of an unstable priority queue is the binary heap used in MiniSat. The split queue present in recent versions of Lingeling (Biere 2015) is only stable with respect to zero and near-zero scores, hence for our purposes we consider it to be stable.

Since in practice the choice of data structure is not particularly consequential (Biere and Fröhlich 2015), whenever we need to we will assume that we use a stable priority queue and make an explicit note of it.

Initialization As we mentioned we assume that when deciding a variable that has never been assigned we set its phase statically to 0 . Other ways to choose an initial phase are with the one-sided Jeroslow-Wang heuristic or at random, but our proof is limited to a static initial phase.

Some ways to pick the initial variable order are to use the same static order as the input, the JW order, or to pick a random order. To make our model more robust we assume the order is random and prove that our result holds except for an exponentially small fraction of initial orders.

\subsection{Preprocessing}

Most solvers use preprocessing techniques in an attempt to simplify their inputs before and even interleaved with the search. While we do not aim to cover all of these techniques, we do not want our formulas to contain pure literals, unit clauses, or similar artefacts that can be easily removed. Therefore we look into the following simple preprocessing rules.

- Self-subsuming resolution (Eén and Biere 2005). If we have clauses $C \vee l$ and $D \vee \bar{l}$, and $C \subseteq D$, then we remove $\bar{l}$ from $D$.

- Bounded variable elimination (Eén and Biere 2005). Resolve all clauses containing $x$ with all clauses containing $\bar{x}$. If the resulting set is not larger, replace the original set.

- Failed literal elimination (Le Berre 2001). If setting a literal $l$ unit propagates a conflict, add the unit clause $\bar{l}$.

- Blocked clause elimination (Järvisalo, Biere, and Heule 2010). If a clause contains a literal $l$ such that resolving $C$ with all clauses containing $\bar{l}$ only yields tautologies, then we remove $C$.

\section{Separation}

The key property that we use from the decision heuristics we study is that they give priority to variables participating in conflicts.

Definition 3.1. We say that a decision heuristic rewards conflicts if a variable that has participated in a conflict is always selected before a variable that has never been assigned.

To prove that concrete heuristics reward conflicts we assume that on a problem of size $n$ the solver uses a floating point type with $\Theta(\log n)$ bits of exponent and runs for $\exp (\mathrm{O}(n))$ many steps.

Lemma 3.2. Stable VSIDS, VMTF, ACIDS, CHB, and Stable LRB reward conflicts.

Proof. Let $x$ be a variable that participated in conflict $t$, and $y$ a variable that has never been assigned. All heuristics we consider satisfy $q(x, s) \geq 0$ and $q(y, s)=0$ for all states $s$, hence for stable decision heuristics it is enough to show that $q(x, t)>0$, while for the rest we have to show that $q(x, s)>0$ for all states $s \geq t$.

It is immediate from the definitions that for stable VSIDS we have $q(x, t) \geq 1$, and for VMTF and ACIDS we have $q(x, s) \geq q(x, t)=t$.

For CHB we have $q(x, s) \geq \alpha \mu / \Delta(x, s)>1 /(20 s)$ for all $s \geq t$, and since $s=\exp (\mathrm{O}(n))$, a floating-point representation can distinguish $1 /(20 s)$ from 0 .

Finally, for stable LRB, even if we cannot guarantee that $q(x, t)>0$, we only need to prove that $x$ is selected before $y$ when both choices are available, and as soon as we reach a state $s>t$ where $x$ is unassigned the score of $x$ is updated to $q(x, s) \geq \alpha / t>1 /(20 t)$.

To exploit this property we craft a formula with an easy part and a hard part, but such that setting variables from the easy part makes the solver find a conflict involving all variables from the hard part.

The formula has 5 types of variables, each type subdivided into $k$ blocks: $X=\bigcup_{j \in k} X_{j}$ with $\left|X_{j}\right|=m$ are hard variables, $Y=\bigcup_{j \in k} Y_{j}$ with $\left|Y_{j}\right|=n$ are easy variables, and $P=\bigcup_{j \in k} P_{k}$ with $\left|P_{j}\right|=m+n, Z=\bigcup_{j \in k} Z_{j}$ with $\left|Z_{j}\right|=n$, and $A=\bigcup_{j \in k} A_{j}$ with $\left|A_{j}\right|=3$ are auxiliary variables. This makes a total of $k(2 m+3 n+3)$ variables. We think of $k$ as a constant and assume that $m=\Theta(n)$.

Next we describe a few gadgets out of which we build the formula. The hard gadget consists of a padded formula, that is given a formula $F$ and a clause $C$, the padded formula $F \vee C$ is $\{D \vee C \mid D \in F\}$.

A pitfall gadget $\Psi\left(y_{1}, y_{2}\right)$ is is the CNF $y_{1} \vee y_{2} \vee \overline{p_{j, 1}}, y_{1} \vee$ $y_{2} \vee \overline{p_{j, 2}}, \ldots, y_{1} \vee y_{2} \vee \overline{p_{j, m+n}}$, where $j$ is the block index of both $y_{1}$ and $y_{2}$. We call $y_{1}$ and $y_{2}$ the trap variables. The goal of a pitfall gadget is to deviate the solver's attention from $Y$ variables towards $X$ variables via $P$ variables: observe that setting $y_{1}=0, y_{2}=0$ leads to a parallel propagation of $P_{j}$.

A pipe gadget $\Pi(y)$ is the CNF $y \vee \bigvee_{i \neq 1} p_{j, i} \vee \overline{x_{j, 1}}, y \vee$ $\bigvee_{i \neq 2} p_{j, i} \vee x_{j, 1} \vee \overline{x_{j, 2}}, \ldots, y \vee \bigvee_{i \neq m} p_{j, i} \vee x_{j, 1} \vee \ldots \vee$ $\overline{x_{j, m}}, y \vee \bigvee_{i \neq m+1} p_{j, i} \vee x_{j, 1} \vee \ldots \vee x_{j, m} \vee \overline{z_{j, 1}}, \ldots, y \vee$ $\vee \bigvee_{i \neq m+n} p_{j, i} \vee x_{j, 1} \vee \cdots \vee x_{j, m} \vee z_{j, 2} \vee \cdots \vee \overline{z_{j, n}}$, where 
$j$ is the block index of $y$. Note that the last clause does not contain $z_{j, 1}$. The goal of a pipe gadget is to follow-up on a pitfall gadget: setting $y$ and all $P_{j}$ variables to 0 leads to a sequential propagation of all variables in $X_{j} \cup Z_{j}$.

A tail gadget $\Delta(y, z)$ is the CNF $\overline{a_{j, 1}} \vee a_{j, 3} \vee \bar{z}, \overline{a_{j, 2}} \vee$ $\overline{a_{j, 3}} \vee \bar{z}, a_{j, 1} \vee \bar{z} \vee \bar{y}, a_{j, 2} \vee \bar{z} \vee \bar{y}$, where $j$ is the block index of both $y$ and $z$. The goal of a tail gadget is to ensure that $Z$ variables do not flip values: if $y=1$, then setting $z=1$ immediately leads to a conflict.

We denote $\Psi_{j}=\left\{\Psi\left(y_{1}, y_{2}\right) \mid\left(y_{1}, y_{2}\right) \in\left(\begin{array}{c}Y_{j} \\ 2\end{array}\right)\right\}, \Pi_{j}=$ $\left\{\Pi(y) \mid y \in Y_{j}\right\}$, and $\Delta_{j}=\left\{\Delta(y, z) \mid(y, z) \in Y_{j} \times Z_{j}\right\}$.

Definition 3.3. The pitfall formula $\Phi(G, \chi, n, k)$ consists of $k$ padded copies of an unsatisfiable Tseitin formula, $\mathrm{Ts}_{j}=$ $\operatorname{Ts}(G, \chi) \vee \bigvee_{z_{i} \in Z_{j}} z_{i}, k$ pitfall gadgets $\Psi_{j}, k$ pipe gadgets $\Pi_{j}, k$ tail gadgets $\Delta_{j}$, and $n / 2$ clauses $\Gamma_{\ell}=\bigvee_{j \in[k]} \overline{y_{j, 2 \ell}} \vee$ $\overline{y_{j, 2 \ell+1}}$, which we refer to as easy clauses.

Note that only easy clauses contain variables in different blocks. The total number of clauses on a graph of degree $d$ is therefore $k \cdot\left((2 m / d) \cdot 2^{d-1}+\left(\left(\begin{array}{l}n \\ 2\end{array}\right)+n\right) \cdot(m+n)+4 n^{2}+n / 2\right)$. There is nothing particularly special about a Tseitin formula, and we could replace it with other hard formulas.

We claim that the formula is not affected by the preprocessing rules we described in Section 2.5.

Lemma 3.4. $\Phi$ is invariant with respect to the $S S R, B V E$, FLE, and BCE preprocessing rules.

We omit the proof for space reasons and move into proving Theorem 1.1. First we show how to build short proofs of pitfall formulas.

Lemma 3.5. There is a resolution refutation of $\Phi(G, \chi, n, k)$ of length $2^{\mathrm{O}\left(k^{2}\right)}+\mathrm{O}\left(k n^{2}(n+m)\right)$.

Proof. For any pair of variables $y_{j, \ell}, y_{j, \ell^{\prime}}$ we can derive the clause $y_{j, \ell} \vee y_{j, \ell^{\prime}}$ in $\mathrm{O}(n)$ steps as follows.

First we derive the clause $y_{j, \ell} \vee p_{j, 1} \vee \cdots \vee p_{j, m+n}$. Let $\Pi\left(y_{j, \ell}\right)=C_{1}, \ldots, C_{m+n}$ be the clauses of the corresponding pipe gadget and let $D \in \mathrm{Ts}_{j}$ be a clause that is not satisfied by setting $X_{j}=0$, that is $D=x_{j, i_{1}} \vee \cdots \vee$ $x_{j, i_{d}} \vee z_{j, 1} \vee \cdots \vee z_{j, n}$. We resolve $D$ with the pipe clauses in reverse, that is, we begin with $D_{m+n}=D$ and at each step from $i=m+n$ to 1 we resolve $D_{i}$ with $C_{i}$ to obtain $D_{i-1}$. Note that $D_{i-1}$ is the same as $C_{i}$ with the last literal removed and the literal $p_{i, j}$ added, and in particular $D_{0}=y_{j, \ell} \vee y_{j, \ell^{\prime}} \vee p_{j, 1} \vee \cdots \vee p_{j, m+n}$.

This allows us to derive the clause $y_{j, \ell} \vee y_{j, \ell^{\prime}}$ in $m+n$ steps simply by resolving $y_{j, \ell} \vee p_{j, 1} \vee \cdots \vee \vee p_{j, m+n}$ with all the clauses in the corresponding pitfall gadget.

Using this procedure we can derive the clauses $y_{j, \ell} \vee y_{j, \ell^{\prime}}$ for $j \in[k]$ and $\left(\ell, \ell^{\prime}\right) \in\left(\begin{array}{c}{[2(k+1)]} \\ 2\end{array}\right)$ in $\mathrm{O}\left(k n^{2}(n+m)\right)$ steps. Together with the first $k+1$ easy clauses $\Gamma_{1}, \ldots, \Gamma_{k+1}$, these form a formula $F$ over $2 k(k+1)=\mathrm{O}\left(k^{2}\right)$ variables. We claim that $F$ is unsatisfiable, therefore there is a resolution refutation of $F$ of length $2^{\mathrm{O}\left(k^{2}\right)}$, proving the lemma.

To prove that $F$ is unsatisfiable we reason by contradiction and assume there is an assignment that satisfies $F$. In order to satisfy all $\Gamma_{1}, \ldots, \Gamma_{k+1}$ clauses at least $k+1$ variables must be set to 0 , hence by the pigeonhole principle there is at least one block $j$ with two variables set to 1 . But this falsifies a clause of the form $y_{j, \ell} \vee y_{j, \ell^{\prime}}$, contradicting that an assignment exists.

We end the section proving the hard part of Theorem 1.1, namely that CDCL requires exponential time to solve pitfall formulas.

Theorem 3.6. There exists $\epsilon>0$ and a family of graphs $G_{n}$ of constant degree such that CDCL with a decision heuristic that rewards conflicts runs for $T$ steps on input $\Phi\left(G_{n}, \chi, n, k\right)$ with $\operatorname{Pr}\left[T \leq 2^{\epsilon m}\right] \leq 2^{-\epsilon n}$.

Before dealing with the formal details, let us think of a simplified scenario and discuss what happens if the two first decisions are $y_{1,1}=0$ and $y_{1,2}=0$. In this case the pitfall gadget $\Psi\left(y_{1,1}, y_{1,2}\right)$ immediately propagates all the $P_{1}$ variables $p_{1,1}=0, p 1,2=0, \ldots, p_{1, m+n}=0$. After this, the pipe gadget $\Pi\left(y_{1,1}\right)$ propagates all the $X_{1}$ variables $x_{1,1}=0, x_{1,2}=0, \ldots, x_{1, m}=0$, in this order. At this point we have a truth value assignment that falsifies the first copy of the Tseitin formula (because we are assigning values to all of the variables of an unsatisfiable formula), so it is only the fact that Tseitin clauses are padded with $Z_{1}$ variables, which are unset, that keeps us from a conflict. But immediately after setting $x_{1, m}$, the same pipe gadget $\Pi\left(y_{1,1}\right)$ propagates all the $Z_{1}$ variables $z_{1,1}=0, z_{1,2}=0, \ldots, z_{1, n}=0$, also in this order, and at this point we reach our first conflict.

Analysing the conflict we see that variables $X_{1} \cup Z_{1} \cup$ $\left\{p_{1, i} \mid i>1\right\} \cup\left\{y_{1,1}\right\}$ are all involved in it, and their score is bumped, hence they are the next in line to be decided. With a case analysis we can prove that no matter to which polarity we choose, once we assign all of these variables we reach another conflict, until we eventually declare the formula unsatisfiable and extract a resolution proof from the execution trace. But since we always assigned variables in the first block, we never used any of the clauses from other blocks or any of the easy clauses that go across blocks, hence our proof is in fact a refutation of the first copy of the Tseitin formula, which requires exponentially many steps.

To prove Theorem 3.6 formally we need to deal with additional variables being decided prior to two $Y_{j}$ variables, thus we introduce the following solver states. In state (a) no conflict has occurred, all assigned variables are set to 0 , no pair of $Y_{j}$ variables is assigned, and for each set $Z_{j}$ and $P_{j}$ at least three variables are unassigned. State (b) is like state (a), except that a pair of $Y_{j}$ variables is assigned. In state (c) variables $X_{j} \cup Z_{j} \cup\left\{p_{j, i} \in P_{j} \mid i>1\right\}$ plus one $y_{j, i}$ variable have participated in a conflict; no variable with an index $j^{\prime} \neq j$ has participated in a conflict; all assigned variables with index $j^{\prime}$ are set to 0 ; no pair of $Y_{j^{\prime}}$ variables is assigned; and for each set $Z_{j^{\prime}}$ and $P_{j^{\prime}}$ at least three variables are unset.

The solver starts in state (a) and, since $\Phi$ is unsatisfiable, ends after reaching a conflict that cannot be resolved. We prove that with high probability the solver moves from state (a) to (b) to (c) and ends at state (c).

Lemma 3.7. When the solver moves away from state (a), it moves to state $(b)$ with probability $1-\exp (-\Omega(n))$.

Lemma 3.8. The solver moves from state $(b)$ to state $(c)$.

Lemma 3.9. The solver never leaves state (c). 
This is enough to prove Theorem 3.6.

Proof of Theorem 3.6. Consider the resolution proof $\pi$ induced by a solver run that moves from state (a) to (b) to (c). We hit $\pi$ with a restriction $\rho$ that sets variables in the following way: $X$ variables remain unset, $Y$ and $P$ variables are assigned to 1 , and $Z$ and $A$ variables are assigned to 0 . Observe that $\rho$ falsifies $\Phi$, so for all we know it could be that $\pi \uparrow_{\rho}$ is trivial, but in fact the only clauses that $\rho$ falsifies are $\Gamma$ clauses, and $(\Phi \backslash \Gamma) \uparrow_{\rho}=\operatorname{Ts}(G, \chi)$. Therefore if we show that $\pi$ never uses $\Gamma$ clauses as axioms then we will have that $\pi \uparrow_{\rho}$ is a refutation of $\operatorname{Ts}(G, \chi)$, hence by Theorem 2.1 it has length $\exp (\Omega(m))$, and by Lemma 2.2 it takes $T=\exp (\Omega(m))$ conflicts to produce.

To prove that $\pi$ never uses $\Gamma$ clauses, by Lemma 2.2 it is enough to to show that $\Gamma$ clauses never unit-propagate or conflict, which is true since each easy clause contains two variables from each set $Y_{j}$, and for all but one of the sets $Y_{j}$ there is only one variable ever assigned, so at least $k-1$ variables of each $\Gamma$ clause remain unset at all times.

We finish the proof of the lower bound by proving the main technical lemmas, skipping Lemma 3.8.

Proof of Lemma 3.7. We begin analysing which clauses might unit propagate. At an (a) state padded Tseitin clauses do not propagate because at least two of the $Z$ variables are unset. The clauses of a pitfall gadget can propagate only if both trap variables are set; otherwise variable $p_{j, i}$ is set to 0 and satisfies the clause. Pipe clauses do not propagate because at least two of the $P$ variables are unset. Tail gadgets do not propagate because literals $\bar{y}$ and $\bar{z}$ are either unset or satisfied, and easy clauses do not propagate because each clause has at least half of its variables unset.

Assume that we are at a stable (a) state and the next action is to decide a variable. If it is an $X$ or $A$ variable, then no propagation happens and the next state is a stable (a) state. If a $Y$ variable $y_{j, \ell}$ is decided and another $Y_{j}$ variable is already assigned, then the next state is a (b) state; otherwise no propagation happens and the next state is a stable (a) state. If a $P$ or $Z$ variable is decided then no propagation happens as long as the variable was not the $m+n-2$-th $P_{j}$ or $(n-2)$-th $Z_{j}$ variable to be assigned.

Therefore it is enough to prove that with high probability we decide a pair of $Y_{j}$ variables before any set of $n-m-2$ $P_{j}$ or $n-2 Z_{j}$ variables are decided. In fact we prove that with high probability we decide $k+1 Y$ variables before any $n-2$ variables in $P \cup Z$, which implies the first event.

Let $E$ be the latter event. Then $\operatorname{Pr}[E]=\operatorname{Pr}[H \geq k+1]$, where $H$ is the random variable measuring the number of $Y$ variables decided among the first $n+k-1$ decided $Y \cup Z$ variables, that is a hypergeometric random variable with a population of $N=k(3 n+m)$, a success population of $K=$ $k n$, and a number of draws of $n^{\prime}=n+k-1$.

Using the tail bound $\operatorname{Pr}\left[H \leq(K / N-t) n^{\prime}\right] \leq$ $\exp \left(-2 t^{2} n^{\prime}\right)$ for the hypergeometric distribution (Chvátal 1979) we obtain a bound for $\operatorname{Pr}[H \leq k]$ of

$$
\exp \left(-2 \cdot\left(\frac{n}{3 n+m}-\frac{k}{n+k-1}\right)^{2} \cdot(n+k)\right)
$$

that is $\exp (-\Omega(n))$.

Proof of Lemma 3.9. At a state of type (c), the set of variables that have been involved in a conflict is bounded below by the set $S_{-}$comprising $X_{j} \cup Z_{j} \cup\left\{p_{i, j} \in P_{j} \mid i>1\right\}$ and a variable $y \in Y_{j}$. The set of variables that have been ever assigned is bounded above by the set $S_{+}$comprising all of $X, P_{j}, Z_{j}$, and $A$, one variable from each set $Y_{j^{\prime}}$, and all but three variables from each set $Z_{j^{\prime}}$ and $P_{j}^{\prime}$. We need to prove that we never reach a conflict where $Y_{j^{\prime}}$ variables participate, and in fact we prove that we never propagate any variable from a $j^{\prime}$ block.

We claim it is enough to prove that (i) setting all of the variables in $S_{-}$always produces a conflict and that (ii) setting all of the variables in $S_{+}$does not propagate any variable with index $j^{\prime}$. Indeed, since the decision heuristic rewards conflicts, all of the variables in $S_{-}$must be assigned before a variable not in $S_{+}$is decided, and by item (ii) no such variable is propagated either. But by item (i), once all of the variables in $S_{-}$are assigned we reach a conflict. After resolving the conflict either part of $S_{-}$is unassigned, or we reach another conflict, or the formula is found unsatisfiable and the solver terminates.

To prove claim (i) we assume that all variables in $S_{-}$are assigned and consider the set of $Z_{j}$ variables. If all variables are set to 0 , then we have that $\left.\mathrm{Ts}_{j}\right\rceil_{Z_{j}=0}$ is an unsatisfiable formula over $X$ variables, all of which are assigned, hence at least one clause of $\mathrm{Ts}_{j}$ is falsified. Thus we can assume that some variable $z \in Z_{j}$ is set to 1 . If $y=1$ then we reach a conflict with one of the clauses of the tail gadget $\Delta(y, z)$, possibly after propagating $a_{1}=1$ and $a_{2}=1$, hence we can assume that $y=0$. If any $P_{j}$ variable is set to 1 then a variable $y^{\prime}$ gets propagated to 1 by the pitfall gadget $\Psi\left(y, y^{\prime}\right)$ and we reach a conflict with the gadget $\Delta\left(y^{\prime}, z\right)$. Therefore we can assume that all $P_{j}$ variables are set to 0 , except possibly for $p_{j, 1}$, which may be unassigned. Let $C$ be the first clause in $\Pi(y)$ that is not satisfied, which exists because $z=1$. If $p_{j, 1}=0$ then $C$ is falsified, otherwise $C$ propagates $p_{j, 1}=1$ and we argue as with other $P$ variables.

To prove claim (ii) observe that only easy clauses share variables across blocks, hence setting all the variables in the $j$-th block does not affect a gadget with variables in the $j^{\prime}$-th block. Easy clauses do not unit propagate since at most one variable out of each $Y_{j^{\prime}}$ set of variables is assigned, so at least $k-1$ variables in each easy clause remain unassigned. As for gadgets with variables in the $j^{\prime}$-th block, since all assigned $j^{\prime}$ variables are set to 0 by the phase saving heuristic, we reuse the analysis of Lemma 3.7 for (a) states.

\section{Experimental Evaluation}

We ran experiments on pitfall formulas using the CaDiCaL 1.0.3 (Biere 2017), Glucose 4.1 (Audemard and Simon 2009), and MapleCOMSPS (Liang et al. 2016c) solvers, representing the VMTF, VSIDS, and CHB and LRB heuristics respectively. We also employed an instrumented version of Glucose that allows choosing a static variable order (Elffers et al. 2018).

In order to work with smaller instances we slightly modify the formula description to allow different numbers of $Y$ 
Table 1: Mean CPU time to solve (s)

\begin{tabular}{lrrrrr}
\hline Formula & Cadical & Glucose & CHB & LRB & Fix \\
\hline $\operatorname{Ts}(45)$ & 3331 & 754 & 621 & 424 & 3600 \\
$\Phi(45,6)$ & 2228 & 1917 & 600 & 2598 & $<1$ \\
$\Phi(45,8)$ & 1963 & 2273 & 607 & 2650 & $<1$ \\
$\operatorname{Ts}(50)$ & 3600 & 3600 & 3600 & 3600 & 3600 \\
$\Phi(50,6)$ & 3600 & 3600 & 3600 & 3600 & $<1$ \\
$\Phi(50,8)$ & 3600 & 3600 & 3600 & 3600 & $<1$ \\
\hline
\end{tabular}

and $Z$ variables. As the base graph for the Tseitin gadget we use a random regular graph of degree 4 and either 45 or 50 vertices. The graphs are chosen so that the Tseitin formula of the smaller graph is solved in a few hundred seconds, while the Tseitin formula of the larger graph cannot be solved in less than one hour. We set the block size of $Y$ and $Z$ variables to 30 and 5 respectively, which we experimentally found to be large enough to make the formulas hard, and use $4,6,8$, and 10 blocks but focus on 6 and 8 since these yield formulas that are hard but not impossible.

We ran the solvers with their default parameters, except that we disabled pre- and inprocessing since these turn out to be counterproductive and experimentally lead to worse runtimes. We turned on VMTF and the unsat option in $\mathrm{Ca}-$ DiCaL, and we disabled the adaptive strategies of Glucose to obtain more consistent results. We chose the pure versions of MapleCOMSPS to avoid interference from other heuristics. The static order decides on $Y, X, Z, P$, and $A$ variables in this order.

To account for different ways to initialize the variable order we shuffled the variables and clauses (but not the polarities) with 32 different seeds and report the aggregated result, assigning a time of $3600 \mathrm{~s}$ to unsolved instances. We do not do any shuffling when running with a fixed order.

We can observe in Table 1 that, as predicted, solvers do not do better than if they had to solve a standalone Tseitin formula, while a fixed variable order is faster by several orders of magnitude.

We also tested the effect of mixing VSIDS with random decisions by running Glucose with a probability of choosing a random variable ranging from 0 to 1 . We find that interleaving a moderate amount of random decisions, say with probability 0.2 , is helpful, but as a side effect the runtime variance increases. Random decisions decrease the average runtime and for instance the hardest formulas $\Phi(50,6)$ and $\Phi(50,8)$ become (barely) solvable.

Finally, we briefly investigate how important the assumption that phases are initialized to 0 is by randomizing the variable polarities. The results are mixed: while Cadical and Glucose tend to perform even worse, some of the harder instances become occasionally solvable within the time limit, and MapleSAT with LRB becomes significantly better.

A formula generator is available as a CNFgen module (Lauria et al. 2017) at doi.org/10.5281/zenodo.3544727, and the full data at doi.org/10.5281/zenodo.3544731.

\section{Concluding Remarks}

We proved that CDCL with conflict-rewarding heuristics does not polynomially simulate resolution. This does not mean we should throw away these heuristics, but it does imply that there is room for improvement. Since random choices help, perhaps one could go a step further and actively choose variables that have been long unused.

Speaking of randomness, it would be interesting to determine whether our formula has short proofs using a random decision heuristic. Note that this does not follow immediately from the result of Atserias, Fichte, and Thurley (2011) because the formula contains long clauses.

Two annoyances of our result are that we need to assume a static initial phase, and in some cases we need to assume a stable data structure. It seems plausible that improving our construction and/or analysis would make the proof work with a random initial phase as well, but to get rid of the stability assumption it appears necessary to ensure that the scores of all variables in the hard part of the formula are periodically refreshed, which would require new ideas.

It would also be interesting to find smaller formulas that exhibit the same properties, so that size does not become a factor that can influence experiments.

\section{Acknowledgements}

I am thankful to Armin Biere for clarifying details about practical implementations of VSIDS, to Jakob Nordström and the participants of the BIRS seminar "Theory and Practice of Satisfiability Solving" (18w5208) for inspiring this research, and to the AAAI reviewers for their suggestions. This work was performed at the Tata Institute of Fundamental Research, supported by the Prof. R Narasimhan postdoctoral award. Experiments were run on resources provided by the Swedish National Infrastructure for Computing (SNIC) at HPC2N.

\section{References}

Atserias, A., and Müller, M. 2019. Automating resolution is NPhard. In Proceedings of the 60th IEEE Symposium on Foundations of Computer Science (FOCS '19).

Atserias, A.; Fichte, J. K.; and Thurley, M. 2011. Clause-learning algorithms with many restarts and bounded-width resolution. Journal of Artificial Intelligence Research 40:353-373. Preliminary version in SAT' 09 .

Audemard, G., and Simon, L. 2009. Predicting learnt clauses quality in modern SAT solvers. In Proceedings of the 21 st International Joint Conference on Artificial Intelligence (IJCAI '09), 399-404.

Beame, P.; Kautz, H.; and Sabharwal, A. 2004. Towards understanding and harnessing the potential of clause learning. Journal of Artificial Intelligence Research 22:319-351. Preliminary version in IJCAI' 03 .

Biere, A., and Fröhlich, A. 2015. Evaluating CDCL variable scoring schemes. In Proceedings of the 18th International Conference on Theory and Applications of Satisfiability Testing (SAT'15), volume 9340 of Lecture Notes in Computer Science, 405-422. Springer.

Biere, A. 2015. Lingeling and friends entering the SAT Race 2015. Technical Report 15/2, FMV Report Series, Institute for Formal Models and Verification, Johannes Kepler University. 
Biere, A. 2017. CaDiCaL, Lingeling, Plingeling, Treengeling, and YalSAT entering the SAT competition 2017. In Proceedings of the SAT Competition 2017: Solver and Benchmark Descriptions, 14-15.

Bonet, M. L.; Esteban, J. L.; Galesi, N.; and Johannsen, J. 2000. On the relative complexity of resolution refinements and cutting planes proof systems. SIAM Journal on Computing 30(5):1462-1484. Preliminary version in FOCS ' 98.

Bonet, M. L.; Buss, S.; and Johannsen, J. 2014. Improved separations of regular resolution from clause learning proof systems. Journal of Artificial Intelligence Research 49:669-703.

Buss, S. R., and Kołodziejczyk, L. 2014. Small stone in pool. Logical Methods in Computer Science 10(2):16:1-16:22.

Chvátal, V. 1979. The tail of the hypergeometric distribution. Discrete Mathematics 25(3):285-287.

Eén, N., and Biere, A. 2005. Effective preprocessing in SAT through variable and clause elimination. In Proceedings of the 8th International Conference on Theory and Applications of Satisfiability Testing (SAT'05), 61-75.

Eén, N., and Sörensson, N. 2004. An extensible SAT-solver. In 6th International Conference on Theory and Applications of Satisfiability Testing (SAT '03), Selected Revised Papers, volume 2919 of Lecture Notes in Computer Science, 502-518. Springer.

Elffers, J.; Johannsen, J.; Lauria, M.; Magnard, T.; Nordström, J.; and Vinyals, M. 2016. Trade-offs between time and memory in a tighter model of CDCL SAT solvers. In Proceedings of the 19th International Conference on Theory and Applications of Satisfiability Testing (SAT '16), volume 9710 of Lecture Notes in Computer Science, 160-176. Springer.

Elffers, J.; Giráldez-Cru, J.; Gocht, S.; Nordström, J.; and Simon, L. 2018. Seeking practical CDCL insights from theoretical SAT benchmarks. In Proceedings of the 27th International Joint Conference on Artificial Intelligence (IJCAI '18), 1300-1308.

Goerdt, A. 1993. Regular resolution versus unrestricted resolution. SIAM Journal on Computing 22(4):661-683.

Hertel, P.; Bacchus, F.; Pitassi, T.; and Van Gelder, A. 2008. Clause learning can effectively P-simulate general propositional resolution. In Proceedings of the 23rd National Conference on Artificial Intelligence (AAAI'08), 283-290.

Järvisalo, M., and Junttila, T. A. 2009. Limitations of restricted branching in clause learning. Constraints 14(3):325-356. Preliminary version in $C P^{\prime} 07$.

Järvisalo, M.; Biere, A.; and Heule, M. 2010. Blocked clause elimination. In Proceedings of the 16th International Conference on Tools and Algorithms for the Construction and Analysis of Systems (TACAS '10), 129-144.

Katebi, H.; Sakallah, K. A.; and Marques-Silva, J. P. 2011. Empirical study of the anatomy of modern SAT solvers. In Proceedings of the 14th International Conference on Theory and Applications of Satisfiability Testing (SAT'11), volume 6695 of Lecture Notes in Computer Science, 343-356. Springer.

Lauria, M.; Elffers, J.; Nordström, J.; and Vinyals, M. 2017. CNFgen: A generator of crafted benchmarks. In Proceedings of the 20th International Conference on Theory and Applications of Satisfiability Testing (SAT '17), volume 10491 of Lecture Notes in Computer Science, 464-473. Springer.

Le Berre, D. 2001. Exploiting the real power of unit propagation lookahead. Electronic Notes in Discrete Mathematics 9:59-80.

Liang, J. H.; Ganesh, V.; Poupart, P.; and Czarnecki, K. $2016 \mathrm{a}$. Exponential recency weighted average branching heuristic for SAT solvers. In Proceedings of the 30th AAAI Conference on Artificial Intelligence (AAAI'16), 3434-3440.

Liang, J. H.; Ganesh, V.; Poupart, P.; and Czarnecki, K. 2016b. Learning rate based branching heuristic for SAT solvers. In Proceedings of the 19th International Conference on Theory and Applications of Satisfiability Testing (SAT '16), volume 9710 of Lecture Notes in Computer Science, 123-140. Springer.

Liang, J. H.; Oh, C.; Ganesh, V.; Czarnecki, K.; and Poupart, P. 2016c. MapleCOMSPS, MapleCOMSPS_LRB, MapleCOMSPS_CHB. In Proceedings of the SAT Competition 2016: Solver and Benchmark Descriptions, 52-53.

Marques-Silva, J. P.; Lynce, I.; and Malik, S. 2009. Conflict-driven clause learning SAT solvers. In Biere, A.; Heule, M.; van Maaren, H.; and Walsh, T., eds., Handbook of Satisfiability, volume 185 of Frontiers in Artificial Intelligence and Applications. IOS Press. chapter 4, 131-153.

Mikša, M., and Nordström, J. 2014. Long proofs of (seemingly) simple formulas. In Proceedings of the 17th International Conference on Theory and Applications of Satisfiability Testing (SAT'14), volume 8561 of Lecture Notes in Computer Science, 121-137. Springer.

Moskewicz, M. W.; Madigan, C. F.; Zhao, Y.; Zhang, L.; and Malik, S. 2001. Chaff: Engineering an efficient SAT solver. In Proceedings of the 38th Design Automation Conference (DAC '01), 530-535.

Mull, N.; Pang, S.; and Razborov, A. A. 2019. On CDCL-based proof systems with the ordered decision strategy. Technical Report 1909.04135, arXiv.org.

Pipatsrisawat, K., and Darwiche, A. 2011. On the power of clauselearning SAT solvers as resolution engines. Artificial Intelligence 175(2):512-525. Preliminary version in $C P$ ' 09 .

Ryan, L. 2004. Efficient algorithms for clause-learning SAT solvers. Master's thesis, Simon Fraser University.

Urquhart, A. 1987. Hard examples for resolution. Journal of the ACM 34(1):209-219.

Urquhart, A. 1995. The complexity of propositional proofs. Bulletin of Symbolic Logic 1(4):425-467.

Van Gelder, A. 2005. Pool resolution and its relation to regular resolution and DPLL with clause learning. In Proceedings of the 12th International Conference on Logic for Programming, Artificial Intelligence, and Reasoning (LPAR '05), volume 3835 of Lecture Notes in Computer Science, 580-594. Springer. 